\title{
Common plasma protein marker LCAT in aggressive human breast cancer and canine mammary tumor
}

\author{
Hyoung-Min Park ${ }^{1,2}$, HuiSu Kim ${ }^{1,2}$, Dong Wook Kim ${ }^{1,2}$, Jong-Hyuk Yoon ${ }^{3}$, Byung-Gyu Kim ${ }^{4}$ E Je-Yoel Cho ${ }^{1,2, *}$ \\ ${ }^{1}$ Department of Biochemistry, BK21 Plus and Research Institute for Veterinary Science, School of Veterinary Medicine, Seoul National \\ University, Seoul 08826, ${ }^{2}$ The Canine Cancer Research Center, Seoul National University, Seoul 08826, ${ }^{3}$ Neurodegenerative Disease \\ Research Group, Korea Brain Research Institute, Daegu 41062, ${ }^{4}$ Center for Genomic Integrity, Institute for Basic Science, UNIST, Ulsan \\ 44919, Korea
}

Breast cancer is one of the most frequently diagnosed cancers. Although biomarkers are continuously being discovered, few specific markers, rather than classification markers, representing the aggressiveness and invasiveness of breast cancer are known. In this study, we used samples from canine mammary tumors in a comparative approach. We subjected 36 fractions of both canine normal and mammary tumor plasmas to highperformance quantitative proteomics analysis. Among the identified proteins, LCAT was selectively expressed in mixed tumor samples. With further MRM and Western blot validation, we discovered that the LCAT protein is an indicator of aggressive mammary tumors, an advanced stage of cancer, possibly highly metastatic. Interestingly, we also found that LCAT is overexpressed in high-grade and lymph-node-positive breast cancer in silico data. We also demonstrated that LCAT is highly expressed in the sera of advanced-stage human breast cancers within the same classification. In conclusion, we identified a possible common plasma protein biomarker, LCAT, that is highly expressed in aggressive human breast cancer and canine mammary tumor. [BMB Reports 2020; 53(12): 664-669]

\section{INTRODUCTION}

Among all the malignant tumors, breast cancer is one of the most frequently diagnosed cancers. In fact, it is the moststudied malignancy in the world (1). Despite the efforts of various researchers, the struggle to understand and cure breast cancer continues on many fronts. Many genes have been identified as biomarkers to further understand breast cancer:

*Corresponding author. Tel: +82-2-800-1268; Fax: +82-2-886-1268; E-mail: jeycho@snu.ac.kr

https://doi.org/10.5483/BMBRep.2020.53.12.238

Received 31 October 2020, Revised 16 November 2020, Accepted 24 November 2020

Keywords: Biomarker, Breast cancer, Comparative medicine, Prognostic, Proteomics whether it is invasive or non-invasive (2), whether it fits a classification, etc. Breast-cancer biomarkers can be organized into three major categories: prognostic, predictive, and pharmacodynamic (3). The most frequently used biomarkers are the prognostic and therapy-decision biomarkers, consisting of tissuebased biomarkers, such as estrogen receptor (ER), progesterone receptor (PgR), and human epidermal growth factor receptor 2 (HER2) (4). Additional protein biomarkers were identified by the improvement of mass spectrometry (MS)-based proteomics technologies, which enabled blood analysis of solid tumors (5). Although sequencing technology has been improved, biomarkers that depict advanced-stage malignancies or cancers that undergo metastasis are much scarcer than are early-stage prognostic biomarkers. The most well-known markers are carcinoma antigen 15-3 (CA-15-3), CA-27/29, and carcinoembryonic antigen (CEA) (6), which indicate data relevant for breast cancer, but still more indicators are needed.

Canine mammary tumors, also known as canine mammary gland carcinomas (MGC), are frequently studied alongside human breast cancer. Not only are they studied because of dogs' biological similarities to humans, but they also provide a wellknown animal model for alternative human breast-cancer investigation (7). However, for cancer-indicating markers, canine prognostic biomarkers are rare. Most are inferred indicators, such as CA 15-3, derived from data in human samples. To further understand and diagnose MGC, we need to find suitable biomarkers that depict stage-wise and aggressive mammary tumors.

In this study we focused on discovering aggressiveness biomarkers of canine mammary tumors using canine normal and cancer plasma samples. After an extensive search of 36 fractions of each sample run in mass spectrometry (MS), we further filtered potential targets by means of MRM data and validated them by Western blot. Once we discovered a suitable biomarker, we used in silico data of human breast cancer to investigate its possibility as a human aggressiveness-indicating biomarker and further validated it in human plasma samples and cell lines. This study will provide a novel aggressiveness biomarker that can be applied to both human and canine malignantcancer patients. 


\section{RESULTS}

To maintain MS quality among samples, we followed a basic three-step process for every canine normal and cancer plasma sample: protein depletion, digestion, and fractionation. We used 12 normal and cancer samples for basic profiling and primary targeting. Plasma protein is not easily acquired in normal protein preprocessing, so we used 36 fractions with 6-plex TMT labels for both sample types (Fig. 1A). Each fraction contained proteins located in various timelines, which allowed a higher yield for comparative analysis. Among the samples, we handpicked cancer subtypes that were diagnosed as aggressive or highly metastatic. Histologically, canine mixed tumors are characterized by the presence of myoepithelial cancer cells inside bone/cartilage mesenchymal cells (8), which are a type of highly developed metastatic cancer. After analysis of LC-MS/MS results, we identified a total of 292 proteins, with 54 proteins more elevated in cancer than in normal plasma (Fig. 1B). Elevated proteins included SERPING1 and SERPINA6, which are known to be increased in canine mammary tumors and are currently recognized markers in human breast-cancer patients (9).

\section{Canine LCAT expression increases in mixed type tumors}

Among the increased proteins, we focused on LCAT, or lecithincholesterol acyltransferase, for several reasons. First, LCAT was the fourth highest elevated protein, with a significant $P$ of 0.03 (Table 1). The other proteins that had higher fold-changes were not statistically significant, since LC-MS/MS intensities can differ because of the sample's natural characteristics, such as age or dog breed. Even though LCAT expression was elevated 1.4-fold, it stood out as being a differentially expressed protein, because, unlike transcriptomes, proteomes can influence the normal state even in slightly increased conditions (10).

Second, LCAT is a highly abundant protein that converts free cholesterol into a more hydrophobic form, which is eventually synthesized into high-density lipoproteins (HDL) that gain mobility to move unidirectionally (11). Highly abundant proteins are much more viable biomarker candidates, since they can be detected with ease.

Third and most interestingly, human LCAT is well known to

A

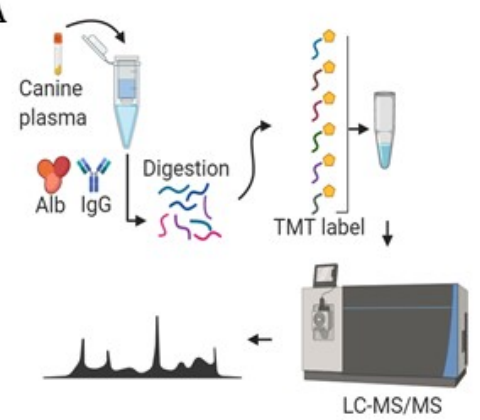

B

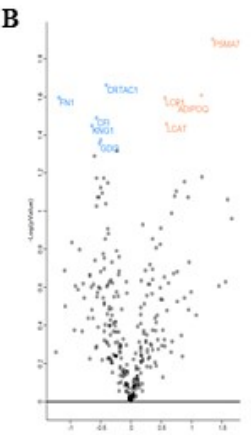

be decreased in overall breast-cancer tissues (12). Because the plasma samples analyzed in our study were highly developed or metastatic carcinomas, this reason alone raised a possibility that LCAT expression patterns may be altered when mammary tumors become more invasive and aggressive. To further address LCAT as a protein highly expressed in mixed tumors, we did additional multi-reaction monitoring (MRM) analysis as a validation. We found that LCAT levels in mixed-tumor cancer were more than 10 times higher than in normal plasma (Fig. 1C). Whereas LCAT was easily detected in mixed tumors, only a small portion was identified in normal samples (Supplementary Fig. 1). Our data showed that the LCAT protein is elevated in the plasma of mixed-type mammary tumors.

Canine mammary tumors can be classified into various types when categorized by histological diagnosis. We further focused on whether LCAT expression is elevated only in mixed tumors or in other cancer subtypes as well. We processed 23 canine plasma samples consisting of normal and distinct cancer subtypes via the three basic steps explained in Fig. 1A. Because of excessive labor, we did three fractions for each sample. Every sample contained $110-230$ proteins with visible LCAT expressions. When we compared normal and cancer specimens, we found that the normal LCAT level was slightly higher (Fig. 2A), which correlates with recent human studies (12). However, when we classified cancer samples into cancer subtypes, mixedtumor samples presented a higher intensity than did simple or complex tumor samples (Fig. 2B). A simple tumor consists of

Table 1. Differential analysis of protein expression in canine plasma

\begin{tabular}{llll}
\hline \multicolumn{1}{c}{$\begin{array}{c}\text { Gene } \\
\text { name }\end{array}$} & $\begin{array}{c}\text { Fold } \\
\text { change }\end{array}$ & $\begin{array}{c}\text { Enriched } \\
\text { sample }\end{array}$ & P-value \\
\hline $\begin{array}{l}\text { PSMA7;PSMA8 } \\
\text { Ig heavy chain V }\end{array}$ & 2.545446 & Cancer & 0.012536934 \\
$\quad$ region MOO & 2.239375 & Cancer & 0.024700876 \\
ADIPOQ & 1.701391 & Cancer & 0.025296237 \\
LCAT & 1.49674 & Cancer & 0.027445789 \\
LCP1;PLS3 & 1.477428 & Cancer & 0.034921527 \\
\hline
\end{tabular}

C

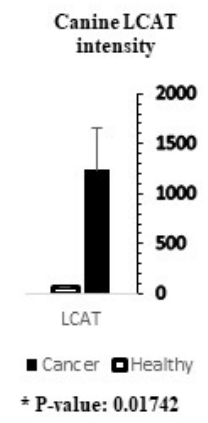

Fig. 1. Proteome profiling of canine normal and cancer plasma samples. (A) Basic schematics of the proteomic procedure. (B) Proteins profiled in each plasma group. Volcano plot indicates top five up-regulated genes (Red) and down-regulated genes (Blue) in each cancer state. (C) Protein LCAT intensity identified by MRM. 
A

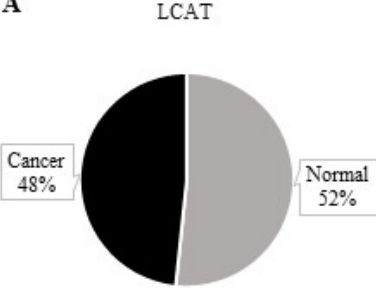

- Normal - Cancer

C

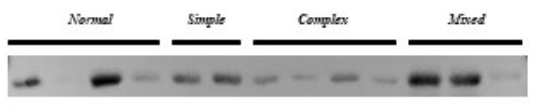

B

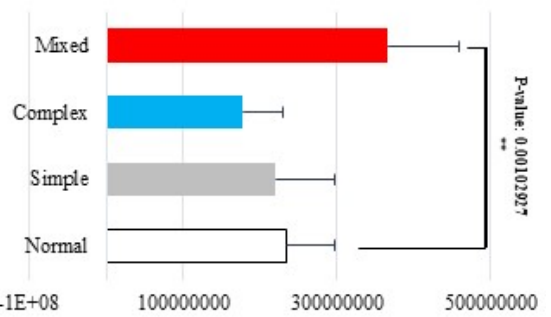

D

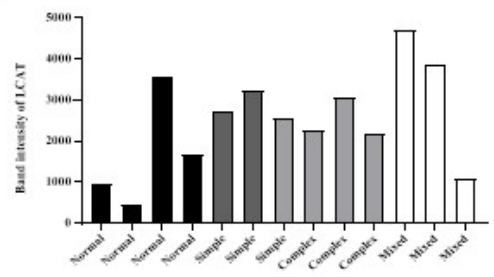

Fig. 2. LCAT expression of canine plasma samples. (A) overall expression comparison of LCAT in 25 samples of normal and cancer plasma. (B) LCAT expression difference between normal and cancer subtypes. (C) Cropped gel indicating LCAT expression of selected canine samples from normal and each cancer subtype. Full-length gels are presented in Supplementary Fig 2. (D) Numeric intensity of LCAT expression derived from Western blot. Samples range from Normal (Black) to Simple (Dark grey), Complex (light grey), and Mixed-type tumors (white).
A
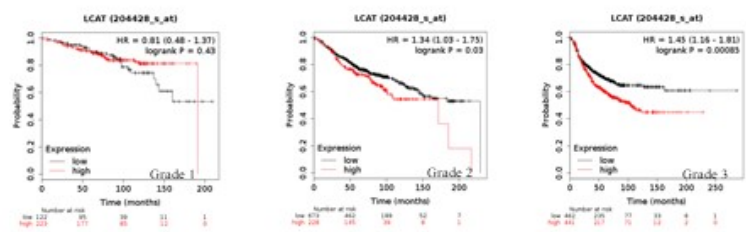

B
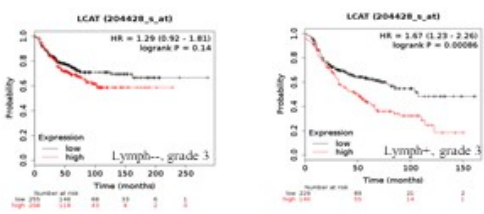

C
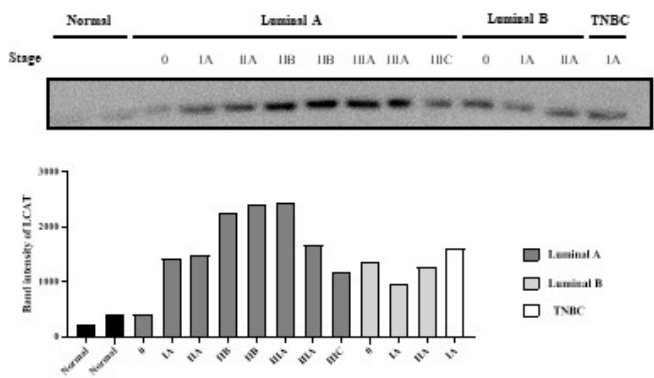

Fig. 3. LCAT expression in human breast cancer. (A) survival rate of breast-cancer patients with grades rang-ng from 1 to 3 . (B) LCAT expression survival rate of breast cancer patients with or without lymphnode metastasis. (C) Cropped gels of Normal, Luminal A, Luminal B, and TNBC subtype breast-cancer patients with different stages indicating LCAT expression. Full-length gels are presented in Supplementary Figure 2. Stages range from the least cancer-like 0 stage to the most aggressive and metastatic IIIC. (D) Intensity of each sample listed in Fig 3C. tubular or papillary adenocarcinomas, usually made up of individual cancer cells derived from their various tissue origins. Complex tumors are microscopically diagnosed by a formation of epithelial and myoepithelial cells. Mixed-type tumors are histologically and microscopically more advanced and metastatic than are other tumor types. Our data further emphasizes that LCAT expression is elevated in a highly developed cancer environment. To further prove the expressive traits of LCAT, we did Western blotting in each group. Using blind selection of normal as well as each cancer subtype, we confirmed that mixed-type tumors tended to have higher levels of LCAT than did normal and other distinct cancer subtypes (Fig. 2C, D). Our results showed that the LCAT protein is elevated in the plasma of highly developed, invasive, and metastatic mammary tumors such as mixed tumors.

Human LCAT expression increases in aggressive cancer states Human LCAT activity is constrained in breast cancer. However, detailed expression profiles of distinct cancer subtypes have not yet been addressed. We analyzed LCAT expression in silico data of human breast cancer tissues provided by the TCGA database. Because such data lack specific stage information, we separated human breast cancer into grades, from lowest, normal-like grade 1 to highly aggressive and invasive grade 3 (Fig. 3A) (13). Survival analysis of each grade presented dissimilar outcomes. Expression levels of LCAT in grades 1 and 2 did not seem to influence the death rate. On the other hand, high expression of LCAT decreased the survival rate of patients with grade 3 breast cancer. This provided evidence that LCAT might have a role in aggressive types of breast cancer. We further sorted grade 3 patients into lymph-node positive and negative types. Lymph nodes can be viable metastasis indicators, since they are one of the most common organs involved in 
aggressive breast-cancer metastasis (14). Interestingly, patients with lymph-node metastasis had a drastic decline in survival rates compared to those of patients with non-metastatic breast cancer (Fig. 3B). Therefore, it was clear that LCAT has a negative correlation with the survival rate of patients with breast cancer that had substantially developed and undergone metastasis.

Survival analysis of breast-cancer patients' data alone does not fully explain whether the higher death rate is caused by differential expression or functional alteration. To validate human LCAT expression in breast-cancer plasma, we selected various subtypes and stages suitable for stage-wise analysis for Western blotting from 24 samples. Surprisingly, for luminal A plasma, LCAT expression was correlated with an increased stage of cancer development (Fig. 3C). Stages of breast cancer ranged from the in situ stage 0 to the very invasive and developed stage 3 . Stage 0 was mainly intact within the ductal parts of the breast, which expressed LCAT patterns similar to those of normal patients. However, when the cancer's characteristics became more aggressive with continuous development, LCAT expression increased. The second stage of breast cancer could be further separated by the possibility of lymph-node metastasis. Stage 2B seemed to be more invasive into the lymph nodes than $2 \mathrm{~A}$ was, as was reflected in the further elevated expression of LCAT (Fig. 3D). This set of data, although tested in only a few samples and needing to be studied with more samples, indicated again that the LCAT plasma level correlates with the aggressiveness of breast cancers.

\section{DISCUSSION}

In this study, we were able to identify a potentially strong protein biomarker as an aggressive breast cancer indicator by means of a comparative approach using canine mammary tumor plasma. Not only are dogs environmentally related to humans, but they are also genetically more highly conserved than are other universal experimental animals, such as mice or rats (15). This aspect gives a different perspective on human breast cancer. Previous studies have used canine mammary tumor as a suitable model for human breast-cancer research to understand certain breast-cancer subtypes that seldom occur but have high mortality rates, such as TNBC and other myoepithelial carcinomas (16) Our group also reported resemblances of canine mammary tumor to human breast cancer in terms of transcriptomic analyses (17). Though canine mammary tumors do not have criteria related to cancer development stages, tumor subtypes are diagnosed by grades to indicate severity. Complex type mammary tumors are mainly graded $1-2$, which indicates relatively stable cancer cells with a good prognosis. Mixed-type tumors are far more metastatic because of the multiple cancer cells related to breast and bones (18). By using developed metastatic canine mixed-tumor plasma, we were able to discover a novel biomarker that could predict of aggressiveness and metastasis of both human breast cancer and canine mammarygland tumors.
Mammary tumor cells secrete certain proteins that can be used to identify their nature. Furthermore, compared to the direct tissue approach, plasma proteins are a much more viable biomarker for both basic and clinical research (19). Despite the provided advantages, most reported plasma protein biomarkers are not easily accessed, because of their low abundance and co-habitation with abundant proteins, such as albumin and various immunoglobulins. Initially by means of extensive plasma sampling using 6-plex TMT labeling and a wide selection of fractions, we identified the protein LCAT in all normal and cancer samples. LCAT is a well-known enzyme that participates in transporting cholesterol (20). To validate the protein as being a more practical biomarker than are other low-abundance proteins, we inspected an additional 24 plasma samples of canine normal and various mammary tumors with a very compact and reproducible method. As a result, we identified LCAT as a selective biomarker highly elevated in mammary tumors that have undergone a series of developments and metastases.

Before investigating LCAT in human breast cancer, we knew about previous studies which mentioned that LCAT activity is decreased in breast-cancer patients. The main LCAT products, lipoproteins $\mathrm{LDL}$ and $\mathrm{HDL}$, may be potential new causes in breastcancer development (21). Decreased LCAT activity leading to low levels of high-density lipoproteins (HDL) was reported in breast-cancer patients before radiotherapy (22). However, more recent reports have described LCAT and HDL levels rising in breast-cancer subjects. A genome-wide study using 164 discrete variants associated with $\mathrm{HDL}, \mathrm{LDL}$, and cholesterol among 101,424 breast-cancer cases and 80,253 controls provided strong evidence that increased HDL may be related to breast-cancer occurrence (23). The controversial debate about lipoproteins in breast cancer may result from not understanding how LCAT activity influences high- and low-density lipoproteins. The dispute also raises the possibility that LCAT expression is not correlated to breast cancer as a whole, but rather only to certain types. Recent reports point out that LCAT expression is also increased in liver cancer. The mechanism related to reverse cholesterol transport of HDL influences the development of liver cancer (24), which indicates that LCAT is not just a biomarker specific to breast cancer, but also has potential in other types of cancer as well. By means of in silico research and Western blot validation, we have demonstrated that the pattern of human LCAT expression levels resemble that of canine LCAT, which was increased in highly progressed breast cancers within the same classified subtypes. These results not only indicate a discovery of a novel protein biomarker in breast cancer, but could also provide further understanding of the lipoprotein pathway that is involved in aggressive breast-cancer development.

In conclusion, this study, using a comparative analysis approach from canine to human cases, reveals the plasma protein LCAT as a biomarker for advanced breast cancer as well as mammary tumors undergoing metastasis. The findings also provide further evidence that canine samples are viable models for human cancer research and for diagnosing clinical samples 
of both dogs and humans.

\section{MATERIALS AND METHODS}

\section{Plasma sampling}

We obtained canine normal and cancer plasma from the Canine Cancer Research Center project (CCRC). No live animals were directly involved in this study. For each sample, $50 \mu \mathrm{l}$ of plasma was used. We did the depletion process by using the multi-affinity removal spin cartridge top 2 depletion kit (Agilent, Cat \# 5188-8825). We concentrated each product by using a speed-vac and added $200 \mu \mathrm{l}$ of HPLC water to dilute it for further processing. Digestion was done via the filter-aided sample preparation protocol (25). We did desalting by using SDB-RPS resin. The initial six canine normal and cancer samples were pooled into three samples. We used 6-plex tandem mass tagging for the normal and cancer samples. We made 36 fractions by using Waters' HPLC columns. The column length was $25 \mathrm{~cm}$, consisting of $\mathrm{C} 18$ with a pore size of $5 \mu \mathrm{m}$ attached to an HPLC separation unit. We put 24 canine plasma samples through identical depletion and digestion processes. We did fractionation and desalting by using the SDP-RPS 3 fraction method. We obtained human normal and cancer plasma from a local hospital that was involved in the CCRC project. No live patients were directly involved in this study. Each sample was processed in the same way as were the canine samples, with a resulting fraction of 3 using the SDP-RPS method.

\section{Mass spectrometry and peptide analysis}

We did proteomics analysis as previously reported by our group (26). Each fraction was identified by a Q-Exactive Orbitrap mass spectrometer located at the Korean Brain Research Institute (KBRI). Additional samples used for validation were identified using the Orbitrap fusion mass spectrometer located at the Ulsan National Institute of Science and Technology (UNIST). Raw data were collected for initial peptide research. We did protein identification using the Maxquant protein search engine (https:// www.maxquant.org/). Major search options were assembled with six minimal peptides, one unique and one razor peptide. Additional modifications included methyl oxidation and N-term acetylation. We analyzed the data by means of the Perseus proteinanalysis tool attached to the Maxquant software. We did differential analysis by sorting cancer proteins expressed at about 1.2fold times those in normal samples. The P cutoff was set to 0.05.

\section{MRM measurements}

We did Multiple Reaction Monitoring (MRM) analysis as previously reported by our group (26). Briefly, we picked identical plasma samples used in the initial protein search for MRM validation. To each desalted peptide product we added $20 \mu \mathrm{l}$ of $0.1 \%$ TFA in HPLC water. Each sample was subjected to 60 min of liquid chromatography (LC). We identified peptide intensity by means of the triple quad 6490. Identified peptides were compared and validated using the skyline software (https://skyline.ms/project/ home/begin.view?).

\section{Kaplan-Meier (KM) plot analysis}

We did survival analysis using a web-based Kaplan-Meier plotter to evaluate the candidate gene and survival rates in more than 3,000 breast-cancer samples. Grades and subtypes were sorted by the options provided within the KM plotter tool. The hazard ratio (HR) was given with $95 \%$ confidence intervals, and log rank P-value was calculated by autoselecting the best cutoff option.

\section{Westem blot}

We did Western blot analysis as previously reported in our laboratory (27). Depleted proteins were dried by a speed vacuum centrifuge and prepared in HPLC grade water. We carried out SDS-PAGE by using a 10\% polyacrylamide gel. LCAT (Abcam, Cambridge, United Kingdom) antibodies were used at a 1:1,000 dilution.

\section{ACKNOWLEDGEMENTS}

This research was supported by the Bio \& Medical Technology Development Program of the National Research Foundation (NRF) funded by the Ministry of Science and ICT (\#2016M3A9B6026771).

\section{CONFLICTS OF INTEREST}

The authors have no conflicting interests.

\section{REFERENCES}

1. Woolston C (2015) Breast cancer. Nature 527, S101

2. Hoag H (2015) Molecular biology: marked progress. Nature 527, S114-115

3. Ulaner GA, Riedl CC, Dickler MN, Jhaveri K, Pandit-Taskar $\mathrm{N}$ and Weber W (2016) Molecular imaging of biomarkers in breast cancer. J Nucl Med 57 Suppl 1, 53S-59S

4. Harris L, Fritsche H, Mennel R et al (2007) American Society of Clinical Oncology 2007 update of recommendations for the use of tumor markers in breast cancer. J Clin Oncol 25, 5287-5312

5. Geyer PE, Holdt LM, Teupser D and Mann M (2017) Revisiting biomarker discovery by plasma proteomics. Mol Syst Biol 13, 942

6. Banin Hirata BK, Oda JM, Losi Guembarovski R, Ariza CB, de Oliveira CE and Watanabe MA (2014) Molecular markers for breast cancer: prediction on tumor behavior. Dis Markers 2014, 513158

7. Salas Y, Marquez A, Diaz D and Romero L (2015) Epidemiological study of mammary tumors in female dogs diagnosed during the period 2002-2012: a growing animal health problem. PLoS One 10, e0127381

8. Dantas Cassali G, Cavalheiro Bertagnolli A, Ferreira E, Araujo Damasceno K, de Oliveira Gamba C and Bonolo de Campos C (2012) Canine mammary mixed tumours: a review. Vet Med Int 2012, 274608

9. de Ronde JJ, Lips EH, Mulder L et al (2013) SERPINA6, BEX1, 
AGTR1, SLC26A3, and LAPTM4B are markers of resistance to neoadjuvant chemotherapy in HER2-negative breast cancer. Breast Cancer Res Treat 137, 213-223

10. Xie X, Zhao J, Xie L et al (2019) Identification of differentially expressed proteins in the injured lung from zinc chloride smoke inhalation based on proteomics analysis. Respir Res 20, 36

11. Dobiasova M and Frohlich JJ (1999) Advances in understanding of the role of lecithin cholesterol acyltransferase (LCAT) in cholesterol transport. Clin Chim Acta 286, 257-271

12. Subbaiah PV, Liu M and Witt TR (1997) Impaired cholesterol esterification in the plasma in patients with breast cancer. Lipids 32, 157-162

13. Rakha EA, Aleskandarany MA, Toss MS et al (2018) Impact of breast cancer grade discordance on prediction of outcome. Histopathology 73, 904-915

14. Rahman M and Mohammed S (2015) Breast cancer metastasis and the lymphatic system. Oncol Lett 10, 1233-1239

15. Lindblad-Toh K, Wade CM, Mikkelsen TS et al (2005) Genome sequence, comparative analysis and haplotype structure of the domestic dog. Nature 438, 803-819

16. Abdelmegeed SM and Mohammed S (2018) Canine mammary tumors as a model for human disease. Oncol Lett 15, 8195-8205

17. Lee KH, Park HM, Son KH, Shin TJ and Cho JY (2018) Transcriptome signatures of canine mammary gland tumors and its comparison to human breast cancers. Cancers (Basel) 10, 317

18. Tavasoly A, Golshahi H, Rezaie A and Farhadi M (2013) Classification and grading of canine malignant mammary tumors. Vet Res Forum 4, 25-30
19. Surinova S, Schiess R, Huttenhain R, Cerciello F, Wollscheid B and Aebersold R (2011) On the development of plasma protein biomarkers. J Proteome Res 10, 5-16

20. Kosek AB, Durbin D and Jonas A (1999) Binding affinity and reactivity of lecithin cholesterol acyltransferase with native lipoproteins. Biochem Biophys Res Commun 258, 548-551

21. Cedo L, Reddy ST, Mato E, Blanco-Vaca F and Escola-Gil JC (2019) HDL and LDL: potential new players in breast cancer development. J Clin Med 8, 853

22. Ozmen HK and Askin S (2013) Lecithin: cholesterol acyltransferase and na $(+)-k(+)$-ATPase activity in patients with breast cancer. J Breast Cancer 16, 159-163

23. Beeghly-Fadiel A, Khankari NK, Delahanty RJ et al (2019) A Mendelian randomization analysis of circulating lipid traits and breast cancer risk. Int J Epidemiol 49, 1117-1131

24. Jiang JT, Xu N and Wu CP (2007) Metabolism of high density lipoproteins in liver cancer. World J Gastroenterol 13, 31593163

25. Wisniewski JR, Zougman A, Nagaraj N and Mann M (2009) Universal sample preparation method for proteome analysis. Nat Methods 6, 359-362

26. Kim YI, Ahn JM, Sung HJ et al (2016) Meta-markers for the differential diagnosis of lung cancer and lung disease. J Proteomics 148, 36-43

27. Cho HM, Kim PH, Chang HK et al (2017) Targeted genome engineering to control VEGF expression in human umbilical cord blood-derived mesenchymal stem cells: potential implications for the treatment of myocardial infarction. Stem Cells Transl Med 6, 1040-1051 